\title{
Multiple endocrine neoplasia type II B with simultaneous papillary microcarcinoma of thyroid gland-a case report
}

\begin{abstract}
Multiple endocrine neoplasia (MEN) type II B is a rare disorder and represent $5 \%$ of multiple endocrine neoplasia type II. It is characterized by medullary thyroid carcinoma, pheochromocytoma and distinctive external morphological features like marfanoid habitus, mucosal neuromas, thickened eyelids, hypertrophied lips, slender body and proximal muscle wasting. Early diagnosis and surgical interventions for medullary thyroid carcinoma and pheochromocytoma results in improvement in long term survival. We report a case of multiple endocrine neoplasia type II B in a 20years old male presented with distinct facial appearance, marfanoid habitus, everted eyelids, thick hypertrophied lips, mucosal neuromas and on and off giddiness, sweating and flushing of face. Clinical evaluation revealed sustained hypertension with episodes of superadded paroxysm and thickened corneal nerve on fundoscopic examination. Ultrasound of neck revealed bilateral thyroid nodules with enlarged regional neck glands and enlarged parathyroid glands. Contrast enhanced computerized scan of abdomen revealed a large left sided adrenal tumor. His serum calcitonin \& serum and urinary catecholamines were raised with marginal elevation of serum calcium. Upper GI endoscopy revealed a vocal cord nodule. The patient underwent left sided radical adrenalectomy and total thyroidectomy three weeks later, with modified bilateral neck dissection together with parathyroidectomy and implantation of one of the parathyroid in the neck. Histopathology of the adrenal tumor was consistent with pheochromocytoma while thyroid gland was detected to harbor bilateral multicentric medullary carcinoma with simultaneous independent foci of papillary microcarcinoma. The patient is on follow up for last 9 yrs and is doing well.
\end{abstract}

Keywords: multiple endocrine neoplasia, pheochromocytoma, papillary microcarcinoma of thyroid, medullary thyroid carcinoma
Volume 2 Issue 5 - 2015

\author{
Sasanka Kumar Barua,TP Rajeev, Dhekial \\ Phukon pranjit kumar, Sarma Debanga, \\ Baruah SJ, Bagchi Puskal Kumar \\ Department of Urology, Guwahati Medical College and Hospital, \\ India
}

Correspondence: Sasanka K Barua, Department of Urology, Gauhati Medical College Hospital, Guwahati, India, Postal Code781032,Tel +91 9864096583, Email sasankagmch@gmail.com

Received: October 24, 2015 | Published: November 23, 2015
Abbreviations: MEN, multiple endocrine neoplasia; RET, rearranged during transfection

\section{Introduction}

Multiple endocrine neoplasia syndromes consist of rare autosomal dominant mutation in genes regulating cell growth. It affects about 1 in 400000 individuals. MEN type II B is a rare disorder and represent 5\% of MEN II cases. It was first described independently by Wagenmann in 1922 and Froboese in 1923. Morrison and Nevin coined the term Wagenmann -Froboese syndrome for this genetic disorder as a tribute to those who first described this entity. ${ }^{1}$

It is characterized by medullary thyroid carcinoma, pheochromocytoma and distinctive external morphological features like marfanoid habitus, mucosal neuromas, thickened eyelids, hypertrophied lips (Figure 1), slender body and proximal muscle wasting. ${ }^{2}$ Early diagnosis and surgical interventions for medullary thyroid carcinoma and pheochromocytoma results in improvement in long term survival.

\section{Case presentation}

A 20years old male presented with distinct facial appearance, marfanoid habitus, everted eyelids, thick hypertrophied lips, mucosal neuromas and on and off giddiness, sweating and flushing of face.
Clinical evaluation revealed sustained hypertension with episodes of superadded paroxysm and thickened corneal nerve on fundoscopic examination. With suspicion of multiple endocrine neoplasia syndrome, ultrasound of neck was performed which revealed bilateral thyroid nodules with enlarged regional neck glands and enlarged parathyroid glands (Figure 2). Contrast enhanced computerized scan of abdomen revealed a large left sided adrenal tumor. His serum calcitonin \& serum and urinary catecholamines were raised with marginal elevation of serum calcium. Upper GI endoscopy revealed a vocal cord nodule. A diagnosis of multiple endocrine neoplasia type II $\mathrm{B}$ was made based on typical external features together with imaging features of neck and adrenal gland.

Fine needle aspiration of thyroid nodule was consistent with medullary thyroid carcinoma. Chromosomal study had shown point mutation in RET proto-oncogene. However none of his relatives or family members had such features. After careful evaluation and adequate control of hypertension, left sided radical adrenalectomy was performed. His intraoperative period was marked by considerable variations in his haemodynamic but postoperative period was uneventful. Three weeks later, total thyroidectomy (Figure 3) with modified bilateral neck dissection was performed together with parathyroidectomy and implantation of one of the parathyroid in the neck. Post operatively he developed tetany, which responded well to intravenous calcium supplementation. 

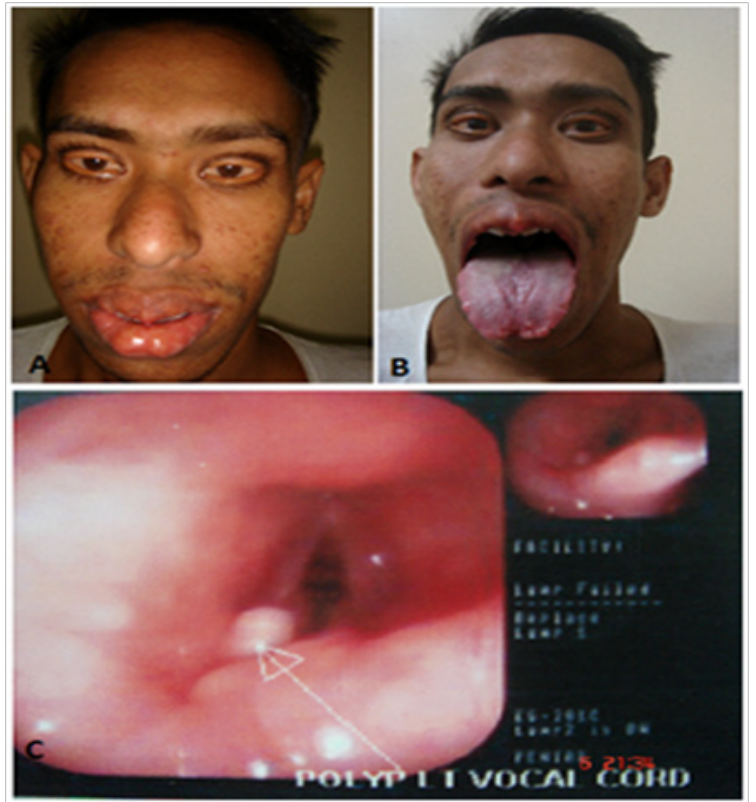

Figure I A. Hypertrophied lip \& eyelids, B. Mucosal Neuromas, C. Upper GI Endoscopy revealed a polyp in left vocal cord.

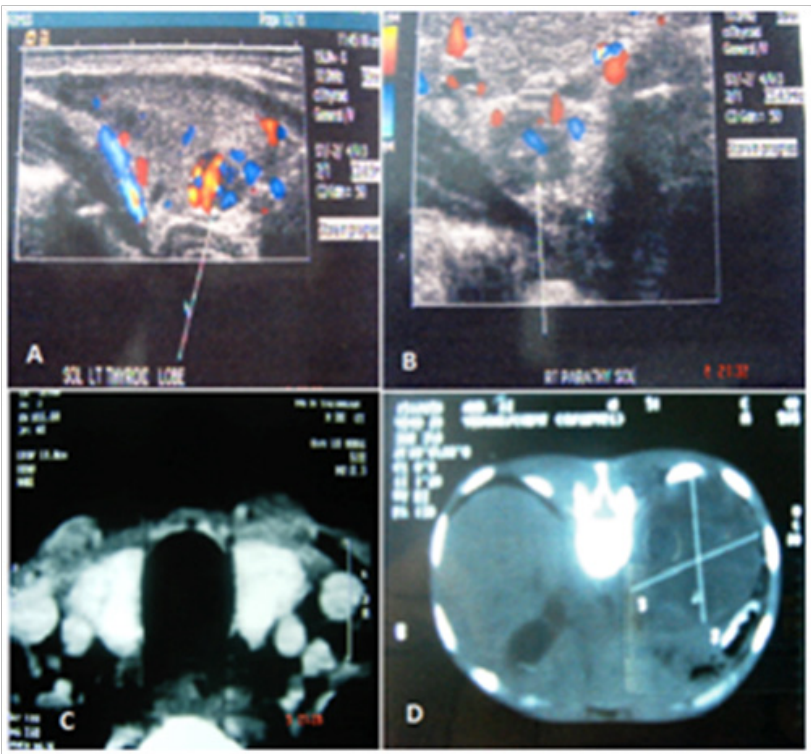

Figure $2 \mathrm{~A}$. Doppler USG showing $0.8 \times 0.7 \mathrm{~cm}$ ill defined lesion with increased vascularity in the right lobe of thyroid gland. B. Doppler USG showing $0.7 \times 0.5$ hypoechoic mass of right parathyroid gland. C. CT scan showing enlarged thyroid gland. D. CT Scan Showing a left adrenal mass.

Figure 4 Histopathology of the adrenal tumor was consistent with pheochromocytoma while thyroid gland was detected to harbor bilateral multicentric medullary carcinoma with simultaneous independent foci of papillary microcarcinoma. Patient was put on oral thyroid hormone and calcium supplementation and a single antihypertensive drug. Patient was discharged at three weeks after thyroid surgery. He was initially followed at three monthly intervals for the first year and six monthly thereafter. The follow up protocol included careful physical examination and estimation of serum calcitonin, calcium, thyroid hormone and catecholamines along with ultrasonography of neck and abdomen. He is currently doing well. His only concern is the external appearance.

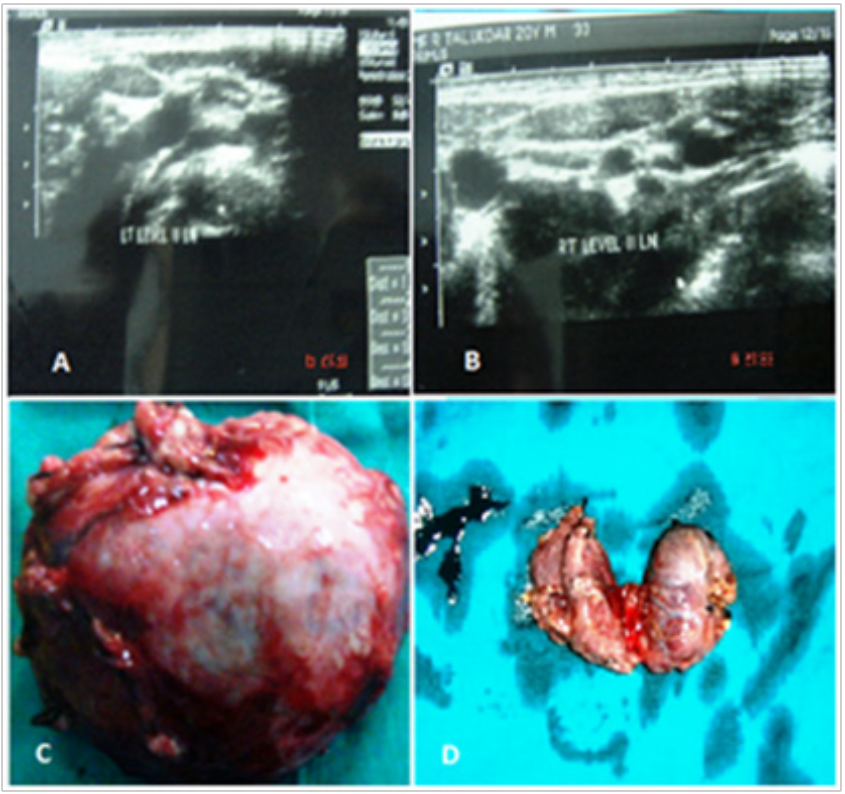

Figure 3 A, B. USG showing enlarged level II neck glands on both side of neck C. Excised specimen of left adrenal tumor. D. Total thyroidectomy specimen.

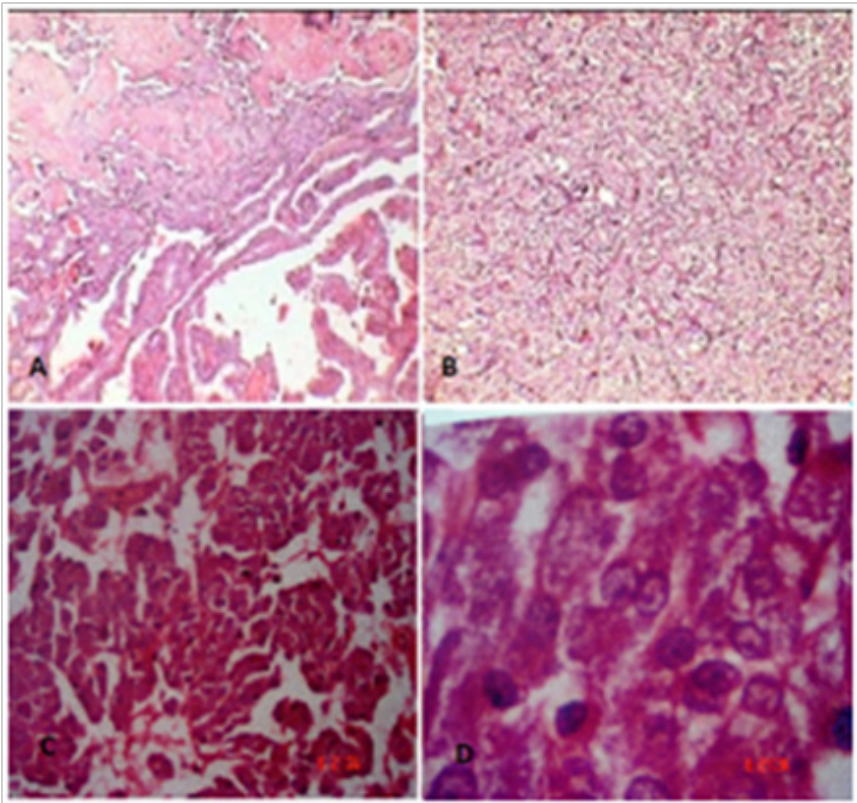

Figure $4 \mathrm{~A}$. Histopathology of the thyroid revealed microcarcinoma. B. Medullary thyroid carcinoma with foci of papillary. C, D. Histopathology of the adrenal tumor suggestive of Pheochromocytoma with internal necrosis.

\section{Discussion}

Multiple endocrine neoplasia type II B is an incompletely penetrant inherited disorder that is transmitted in a dominant fashion with $50 \%$ chance of inheritance while some generation may be spared. ${ }^{3}$ It is primarily caused by a methionine to threonine substitution residue 918 in the kinase domain of the RET receptor; however the molecular mechanism that leads to the typical external appearance is unclear. ${ }^{4}$ More than $95 \%$ cases are associated with specific point mutation in RET proto oncogenes and rarely there may not be any family history of such disorder ${ }^{5}$ as seen in our patient. 
The typical external morphological features like marfanoid habitus, mucosal neuromas, thickened eyelids, mild ptosis, and hypertrophied lips are often associated with multiple endocrinopathies like parathyroid hyperplasia, medullary thyroid carcinoma and Pheochromocytoma. Although prophylactic thyroidectomy is advocated during first year of life, ${ }^{6}$ many with MENII B are diagnosed during adulthood. In such cases pheochromocytoma should be removed prior to total thyroidectomy to avoid intraoperative catecholamine crisis. $^{7}$ The treatment of parathyroid hyperplasia consists of subtotal parathyroidectomy with preservation of a well vascularized portion of one of the gland in situ or its transplantation to the non dominant forearm. ${ }^{3}$

Our patient had some unusual characteristics like absence of family history, presence of parathyroid hyperplasia and independent foci of papillary microcarcinoma together with medullary carcinoma of thyroid, which is extremely rare in multiple endocrine neoplasia type II B syndrome. Simultaneous occurrence of medullary and papillary carcinoma of thyroid is an extremely rare phenomena in multiple endocrine neoplasia syndromes and only reported to occur in multiple endocrine neoplasia type II $\mathrm{A}^{8}$ but such occurrence is never been reported in MENII B cases. Concurrent medullary and papillary carcinoma represent $<1 \%$ of all thyroid malignancies and outcome of MTC did not appear to be influenced by the presence of papillary thyroid cancer. ${ }^{9}$

Patient with multiple endocrine neoplasia type II B diagnosed in adulthood should undergo surgery for extirpation of pheochromocytoma and total thyroidectomy with bilateral lymphadenectomy as early as possible. They need lifelong thyroid hormone supplementation. Those undergoing parathyroidectomy needs additional calcium and vitamin D supplementation. Lifelong follow up is mandatory as metastatic or recurrent medullary carcinoma of thyroid is a cause of mortality even after many years of total thyroidectomy ${ }^{5}$ and extra adrenal or contra lateral pheochromocytoma can occur later in adult with multiple endocrine neoplasia type II B. Although a genetic screening of the family members is obligatory, due to cost constraint it was not advocated in our case.

\section{Summary}

Multiple endocrine neoplasia is an extremely rare genetic disorder characterized by typical cutaneous manifestation and external appearance along with endocrine malignancies. In our case all the external manifestations and internal endocrinopathies were quite evident, that lead us to early interventions with acceptable outcome. Prompt diagnosis and removal of pheochromocytoma and medullary thyroid carcinoma improves survival even if it is diagnosed in adults. However lifelong endocrine supplementations with thyroid hormone and calcium are necessary to prevent morbidity. Periodic follow up is mandatory to detect recurrence or metastases from medullary carcinoma of thyroid and contra lateral or extra adrenal pheochromocytoma in the long run. A multidisciplinary approach is needed for acceptable outcome after interventions.

\section{Acknowledgements}

None.

\section{Conflict of interest}

The author declares no conflict of interest.

\section{References}

1. Morrison PJ, Nevin NC. Multiple endocrine neoplasia type IIB (Mucosal Neuromas Syndrome or Wagenmann-Froboese syndrome). $J$ Med Genet. 1996;33(9):779-782.

2. Gorlin RJ, Vickers RA. Multiple mucosal neuromas, pheochromocytoma, medullary carcinoma of thyroid and marfanoid body builds with muscle wasting: Re examination of a syndrome of neural crest malmigration. Birth Defect Oriq Artic Ser. 1971;7(6):69-72.

3. Hashmati HM, Hofbauer LC. Multiple endocrine neoplasia type 2:recent progress in diagnosis and management. Eur $J$ Endocrinol. 1997;137(6):572-578

4. Gujral TS, Singh VK, Jia Z, et al. Molecular mechanism of RET receptor mediated oncogenesis in Multiple endocrine neoplasia type II B. Cancer Res. 2006;66(22):10741-10749.

5. Nakata S, Okugi H, Saitoh Y, et al. Multiple endocrine neoplasia type II B. Int J Urol. 2001;8(7):398-400.

6. van Heurn LW, Schaap C, Sie G, et al. Predictive DNA testing for MEN 2:A therapeutic challenge of prophylactic thyroidectomy in very young children. J Pedatr Surg. 1999;34(4):568-571.

7. Lee NC, Norton JA. Multiple endocrine neoplasia type II B, genetic basis and clinical expression. Surg Oncol. 2000;9(3):111-118.

8. Giacomelli L, Guerriero G, Falvo L, et al. Simultaneous occurrence of medullary carcinoma and papillary micro carcinoma of thyroid in a patient with MEN IIA syndrome, report of a case. Tumori. 2007;93(1):109-111.

9. Biscolla RP, Ugolini C, Sculli M, et al. Medullary and papillary tumors are frequently associated in same thyroid gland without evidence of reciprocal influence in their biologic behavior. Thyroid. 2004;14(11):946-952. 